\title{
On the interpretation of negative birefringence observed in strong-field optical pump-probe experiments: high-order Kerr and plasma grating effects
}

\author{
G. Karras, P. Béjot, J. Houzet, E. Hertz, F. Billard, B. Lavorel, and O. Faucher \\ Laboratoire Interdisciplinaire Carnot de Bourgogne (ICB), UMR 6303 CNRS-Université de Bourgogne, \\ 9 Av. A. Savary, BP 47870, F-21078 DIJON Cedex, France
}

(Dated: July 19, 2018)

\begin{abstract}
The analysis of negative birefringence optically induced in major air components (Loriot et al., [1, 2]) is revisited in light of the recently reported plasma grating-induced phase-shift effect predicted for strong field pump-probe experiments (Wahlstrand and Milchberg, [3]). The nonlinear birefringence induced by a short and intense laser pulse in argon is measured by femtosecond time-resolved polarimetry. The experiments are performed with degenerate colors, where the pump and probe beam share the same spectrum, or with two different colors and non-overlapping spectra. The interpretation of the experimental results is substantiated using a numerical $3 \mathrm{D}+1$ model accounting for nonlinear propagation effects, cross-beam geometry of the interacting laser pulses, and detection technique. The model also includes the ionization rate of argon and high-order Kerr indices introduced by Loriot et al. enabling to assess the contribution of both terms to the observed effect. The results show that the ionization-induced phase-shift has a minor contribution compared to the high-order Kerr effect formerly introduced, the latter allowing a reasonably good reproduction of the experimental data for the present conditions.
\end{abstract}

PACS numbers: 42.65.Jx,42.65.Hw,42.65.Re

\section{INTRODUCTION}

Recently, we reported on the observation of unexpected large nonlinear dynamics in the measurements of optically induced birefringence of major air components using a pump-probe time-resolved polarization technique [1, 2]. For the investigated $\mathrm{N}_{2}, \mathrm{O}_{2}$, and $\mathrm{Ar}$ gases, the intensity dependence of the reported effect shared the same behavior. In the lower limit, i.e., below $\sim 10 \mathrm{TW} / \mathrm{cm}^{2}$, the induced birefringence followed the applied field intensity. Around $25 \mathrm{TW} / \mathrm{cm}^{2}$, a saturation of the signal was observed leading to an inversion and then followed by a very fast change of the birefringence for larger intensities. We attributed the transition between these linear and nonlinear regimes to a saturation of the electronic Kerr effect induced by the intense short laser pulse. Based on different assumptions, we proposed a phenomenological model by introducing in the standard definition of the nonlinear refractive index $n_{\text {Kerr }}=n_{2} I$, where $n_{2}$ is the Kerr index and $I$ is the applied intensity [4], high-order Kerr (HOK) terms $n_{4}, n_{6}, n_{8}$, and eventually $n_{10}[1,2,2,5]$. Starting from the weak field regime, each term was successively fitted to the experiment by gradually increasing the intensity so as to minimize cross correlations. The intensity at the interaction region was carefully estimated by simultaneously recording the retarded birefringence signal resulting from field-free alignment of a molecular gas [6 8]. In the HOK model, where the nonlinear refractive index is defined by $n_{\text {Kerr }}=n_{2} I+n_{4} I^{2}+n_{6} I^{3}+n_{8} I^{4}$, the saturation and inversion of the electronic Kerr index are due to negative $n_{4}$ and $n_{8}$ terms. It is well known that the optical Kerr effect plays a central role in the nonlinear propagation of short and intense laser pulses such as selfphase modulation, self-focusing, pulse compression, and laser filamentation $9-13$. The potential impact of the
HOK model on the last process was presented in Refs. [14] and [5]. These works reveal that Kerr saturation due to high-order terms can lead under certain conditions to a self-guided nonlinear pulse propagation without requiring the need for a plasma production. This prediction stands against the standard model of filamentation where the plasma acts as the primary mechanism responsible for the arrest of Kerr-induced self focusing in a gas. It is important to mention that the HOK model predicts also the standard regime of filamentation; the boundary between the standard and the all-Kerr regime being ruled by the characteristics of the applied laser electric field [5]. The existence of these two distinguishable filamentation regimes was supported by an experiment 15 that compared long pulse versus short pulse filamentation produced in argon using a bipulse setup.

The change of paradigm introduced by the HOK model [16] has ignited an ongoing active debate questioning the physical meaning or the existence of the Kerr saturation reported in Refs. [1, 2]. In this respect, several theoretical studies have been focused on the microscopic origin of the HOK effect either by solving the $1 \mathrm{D}$ or $3 \mathrm{D}$ Schrödinger equation for an atom exposed to a strong laser field [17 24] or by applying the concept of nonlinear Kramers-Kronig relations 25, 26]. Although the literature published on this issue has contributed to get a much better insight into the temporal dynamics responsible for the nonlinear atomic response at the driving field frequency, the relevance of the HOK model for shortpulse long-wavelength propagation is still controversial. The existence of the HOK effect has also been challenged by the recent report of Wahlstrand and Milchberg on ionization-induced birefringence in pump-probe experiments [3]. The authors of this work argue that a pumpprobe cross-phase shift generated through a plasma grat- 
ing $(\mathrm{PG})$ could provide an alternative explanation to the negative birefringence observed in Refs. [1, 2]. In more details, they show that in case of non-collinear pumpprobe experiment employing degenerate frequencies (i.e., the same color for the pump and probe beam) and noncollinear linearly polarized fields, the optical interference between the pump and probe field leads to a spatial intensity grating. When the pump intensity is sufficiently high, the ionization induced by this intensity modulation embeds a plasma grating into the gas medium. Because the grating is due to the addition of the pump field with the projection of the probe field along the direction of the pump field, the resulting two-beam coupling effect only affects the phase of the parallel field components. This leads to a transient birefringence undergone by the probe beam during the temporal overlapping of the two beams. The pump-probe signal predicted by the plasma grating is featured by an asymmetric profile as well as a power dependence proportional to the ionization rate of the gas [3, 27]. The former lies in the fact that the plasma stems from the accumulation of ionized electrons during the pulse and therefore behaves as a retarded effect.

The aim of the present work is to assess the role of the plasma grating on the negative birefringence signal observed by Loriot et al. in a pump-probe experiment [1, 2] . To this end, we will present a quantitative analysis of a new series of experimental data based on $3 \mathrm{D}+1$ numerical simulations including both semi-empirical HOK and PG models. By investigating the influence of the pulse parameters like the pump intensity, frequency chirp, and probe frequency we will show that the negative birefringence observed in the experiment can not be solely ascribed to the plasma grating effect. The experimental setup used for the one- and two-color transient birefringence measurements is discussed in section [II The theoretical model used for the numerical simulations of the $3 \mathrm{D}+1$ nonlinear propagation in a pump-probe crossbeam geometry is described in section III The results of the one- and two-color experiment together with direct comparisons with numerical simulations are presented and discussed in section IV]

\section{EXPERIMENTAL SETUP}

The experimental setup used for the presented measurements is depicted in Fig. 10 It is a typical pumpprobe scheme that has been reported previously [1] and takes advantage of the depolarization of the probe beam when the later interacts with a birefringent medium optically induced by a pump pulse. The laser source is a chirped pulse amplified Ti:Sapphire system which delivers pulses centered at $790 \mathrm{~nm}$ with $3 \mathrm{~mJ}$ energy per pulse (100 fs pulse duration) at $1 \mathrm{kHz}$ repetition rate. Pump and probe pulses are formed by splitting the output beam into two parts using a beam splitter. Control of the energy and polarization of each pulse is accomplished using zero-order half-wave plates and glan polarizers, while

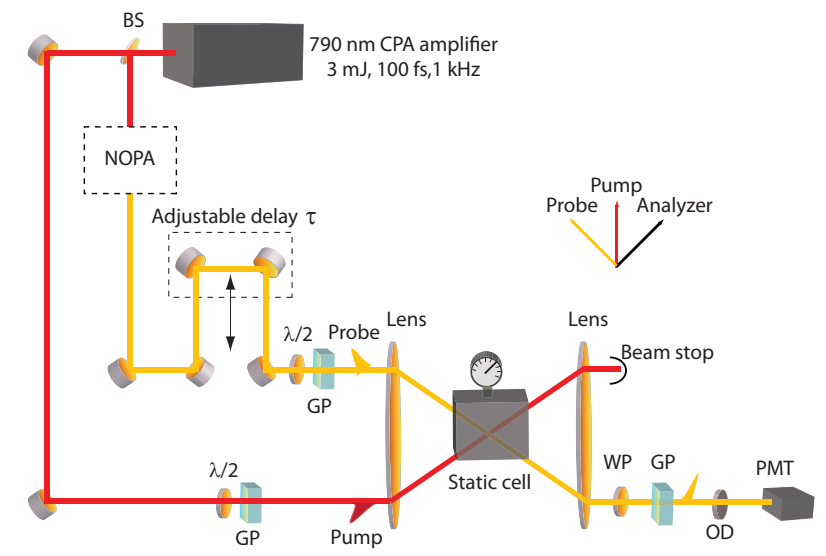

FIG. 1: (Color online) Pump-probe setup for laser-induced birefringence measurements. BS: Beam Splitter, GP: Glan Polarizer, OD: Neutral Optical Density, WP: Wave Plate, PMT: Photo Multiplier Tube. The relative polarizations of the pump, probe, and analyzer are shown in the inset. The noncollinear optical parametric amplifier (NOPA) is only used for the purpose of the two-color pump-probe measurements.

their relative delay is adjusted using a motorized stage. Wavelength tunability, here for the probe beam, whenever needed is attained by directing the beam to a noncollinear optical parametric amplifier (NOPA). The intensity ratio of the probe over the pump beam is less than $1 \%$ and they are both linearly polarized at $45^{\circ}$ relative to each other. Both beams are overlapped at small angle $\left(3^{\circ}\right)$ and focused in the center of a $27 \mathrm{~cm}$-long static cell with a $\mathrm{f}=30 \mathrm{~cm}$ plano-convex focal lens resulting in beam waists of $40 \mu \mathrm{m}$. The gas used for the reported data is Ar and the pressure in the cell is kept low in order to minimize the contribution from nonlinear propagation effects. The depolarized probe beam after the cell passes through a linear crossed analyzer and the signal is measured using a photomultiplier and sampled by a boxcar integrator. This homodyne signal carries the information about the transient induced birefringence and can be written as

$$
\mathcal{S} \propto \int_{-\infty}^{\infty} I_{\text {probe }}(t-\tau)\left[\Delta n\left(t, I_{\text {pump }}\right)\right]^{2} d t,
$$

where $\Delta n$ is the difference in the refractive index along and perpendicular the laser polarization axis and $I_{\text {probe }}$ ( $\left.I_{\text {pump }}\right)$ is the intensity of the probe (pump) beam. From the above equation we see that the sign of the transient birefringence is not revealed in this case due to the quadratic dependence of the signal on $\Delta n$. In order to gain access to the sign of this transient birefringence we add a local oscillator (L.O.) using an adjustable wave plate. The amplitude of this permanent birefringence is adjusted by changing the applied mechanical stress and the sign is controlled by rotating the wave plate perpendicular to the propagation direction by $90^{\circ}$ [28, 29]. The 
detected signal in this case is given by

$$
\mathcal{S}_{ \pm} \propto \int_{-\infty}^{\infty} I_{\text {probe }}(t-\tau)\left[\Delta n\left(t, I_{\text {pump }}\right) \pm \text { L.O. }\right]^{2} d t
$$

where \pm denotes the sign of the L.O. Subtraction of the two experimental traces for positive and negative L.O. results to the pure heterodyned signal:

$$
S_{\text {heterodyne }} \propto\left(\mathcal{S}_{+}-\mathcal{S}_{-}\right) .
$$

As discussed in section [V] this procedure allows to prevent the detection of possible energy transfer or dichroism.

\section{NUMERICAL MODEL}

The equations system driving the propagation of the global electric field envelopes $\mathcal{E}_{x}\left(\mathcal{E}_{y}\right)$ polarized along $x$ (y) read in the reciprocal space [30]:

$$
\begin{aligned}
\partial_{z} \widetilde{\mathcal{E}}_{x}= & i\left(k_{z}-k_{1} \omega\right) \widetilde{\mathcal{E}}_{x}+\frac{1}{k_{z}}\left(\frac{i \omega^{2}}{c^{2}} \widetilde{P}_{x, \mathrm{NL}}-\frac{\omega}{2 \epsilon_{0} c^{2}} \widetilde{J}_{x}\right) \\
& -\widetilde{L}_{x, \text { losses }}, \\
\partial_{z} \widetilde{\mathcal{E}}_{y}= & i\left(k_{z}-k_{1} \omega\right) \widetilde{\mathcal{E}}_{y}+\frac{1}{k_{z}}\left(\frac{i \omega^{2}}{c^{2}} \widetilde{P}_{y, \mathrm{NL}}-\frac{\omega}{2 \epsilon_{0} c^{2}} \widetilde{J}_{y}\right) \\
& -\widetilde{L}_{y, \text { losses }},
\end{aligned}
$$

where $\widetilde{P}_{\mathrm{NL}}$ is the nonlinear polarization, $\widetilde{J}$ is the plasmainduced current, $\widetilde{L}$ accounts for nonlinear losses expressed in the reciprocal space, and

$$
\begin{aligned}
k(\omega) & =n(\omega) \omega / c \\
k_{z} & =\sqrt{k^{2}(\omega)-\left(k_{x}^{2}+k_{y}^{2}\right)} \\
P_{x, \mathrm{NL}} & =n_{2}\left|\mathcal{E}_{x}\right|^{2} \mathcal{E}_{x}+\frac{2}{3} n_{2}\left|\mathcal{E}_{y}\right|^{2} \mathcal{E}_{x}+\Delta n_{x, \mathrm{HOK}} \mathcal{E}_{x} \\
P_{y, \mathrm{NL}} & =n_{2}\left|\mathcal{E}_{y}\right|^{2} \mathcal{E}_{y}+\frac{2}{3} n_{2}\left|\mathcal{E}_{x}\right|^{2} \mathcal{E}_{y}+\Delta n_{y, \mathrm{HOK}} \mathcal{E}_{y}, \\
\widetilde{J}_{x, y} & =\frac{e^{2}}{m_{\mathrm{e}}} \frac{\nu_{\mathrm{e}}+i \omega}{\nu_{\mathrm{e}}^{2}+\omega^{2}} \widetilde{\mathcal{E}}_{x, y} \\
\partial_{t} \rho & =\sigma_{N}\left(\left|\mathcal{E}_{x}\right|^{2 N}+\left|\mathcal{E}_{y}\right|^{2 N}\right)\left(\rho_{\mathrm{at}}-\rho\right) \\
L_{x, \text { losses }} & =\frac{N \hbar \omega_{0} \sigma_{N} \rho_{\mathrm{at}}}{2}\left|\mathcal{E}_{x}\right|^{2 N-2} \mathcal{E}_{x} \\
L_{y, \text { losses }} & =\frac{N \hbar \omega_{0} \sigma_{N} \rho_{\mathrm{at}}}{2}\left|\mathcal{E}_{y}\right|^{2 N-2} \mathcal{E}_{y}, \\
\Delta n_{x, \mathrm{HOK}} & =\sum_{j=2}^{5} n_{2 j}\left(\left|\mathcal{E}_{x}\right|^{2 j}+\frac{j+1}{2 j+1}\left|\mathcal{E}_{y}\right|^{2 j}\right) \\
\Delta n_{y, \mathrm{HOK}} & =\sum_{j=2}^{5} n_{2 j}\left(\left|\mathcal{E}_{y}\right|^{2 j}+\frac{j+1}{2 j+1}\left|\mathcal{E}_{x}\right|^{2 j}\right)
\end{aligned}
$$

where $\omega$ is the angular frequency, $n$ is the frequency dependent refractive index, $c$ is the light velocity, $k(\omega)$ is

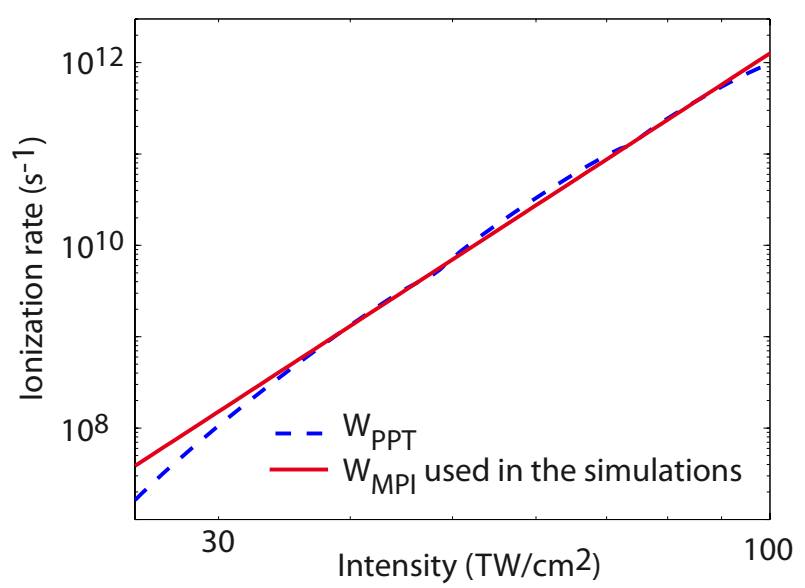

FIG. 2: (Color online) Ionization rate at $800 \mathrm{~nm}$ calculated with the PPT formulation (blue dashed line) as a function of intensity and the effective multiphoton rate following a Nthorder $(N=7.5)$ intensity power law used in the present work (red solid line).

the frequency dependent wavenumber, $k_{1}=\partial k / \partial \omega \mid \omega_{0}$ corresponds to the inverse of the group velocity, $k_{x}$ and $k_{y}$ are the conjugate variables of $x$ and $y$ in the reciprocal space, $m_{\mathrm{e}}$ and $e$ are the electron mass and charge respectively, $n_{2}$ is the nonlinear refractive index, $\rho\left(\rho_{\text {at }}\right)$ is the electron (atom) density, $N$ is the ionization nonlinearity, $\sigma_{N}$ is the ionization cross-section, and $\nu_{\mathrm{e}}$ is the effective collision frequency (see Table [). In Eqs. 6] and 7, $n_{2 j}$ are the high-order Kerr terms measured in [1, 2].

\begin{tabular}{|c|c|c|c|}
\hline $\mathrm{n}_{2}$ & $\mathrm{~N}$ & $\sigma_{N}$ & $\nu_{e}$ \\
\hline $10^{-7} \mathrm{~cm}^{2} / \mathrm{TW} / \mathrm{bar}$ & & $10^{-3} \mathrm{TW}^{-N} \mathrm{~cm}^{2 N} / \mathrm{s} / \mathrm{bar}$ & $\mathrm{fs}^{-1} / \mathrm{bar}$ \\
\hline 1 & 7.5 & 1.26 & 190 \\
\hline
\end{tabular}

TABLE I: Physical parameters used in the model: Kerr index $n_{2}$ [2], nonlinearity $N$ and ionization cross-section $\sigma_{N}$ [31, 32], and effective collision frequency $\nu_{e}$ of Ar at $800 \mathrm{~nm}$.

The nonlinear refractive index expansion given in Eqs. 6 and 7 is consistent with the expression that has been used in Refs. [1, 2] in order to define the $n_{2 j}$ values from the birefringence measurements. This expression relies on the relation between the parallel and perpendicular component of the two first terms $n_{2}$ and $n_{4}$ derived by Arabat and Etchepare 33] and generalized at any order by following the same approach [1]. More recently, an analytical derivation including up to the $n_{8}$ term has shown that are our formula is not strictly valid, although the error introduced on the reported high-order Kerr terms is only a few percent [34] (which remains within the experimental error of our measurements). For consistency, since the present birefringence calculations are based on the Kerr index values reported in Ref. [2], we use the same expansion as previously.

For a pump and probe field time delayed by $\tau$ and linearly polarized as depicted in Fig. 1, the initial electric 
field writes:

$$
\begin{aligned}
\mathcal{E}_{x}(z=0) & =G(y)\left[\mathcal{E}_{\text {pump }} F_{\text {pump }}(t) H_{\text {pump }}(x)+\right. \\
& \left.+\frac{1}{\sqrt{2}} \mathcal{E}_{\text {probe }} F_{\text {probe }}(t-\tau) H_{\text {probe }}(x)\right] \\
\mathcal{E}_{y}(z=0) & =\frac{1}{\sqrt{2}} \mathcal{E}_{\text {probe }} F_{\text {probe }}(t-\tau) G(y) H_{\text {probe }}(x)(9)
\end{aligned}
$$

with

$$
\begin{aligned}
G(y) & =e^{-\frac{y^{2}}{\sigma_{y}^{2}}} e^{-i k_{0}\left(R_{z}-\sqrt{R_{z}^{2}-y^{2}}\right)}, \\
H_{\text {pump }}(x) & =e^{-\frac{\left(x+x_{0}\right)^{2}}{\sigma_{x}^{2}}} e^{i \Phi_{\text {pump }}(x)}, \\
H_{\text {probe }}(x) & =e^{-\frac{\left(x-x_{0}\right)^{2}}{\sigma_{x}^{2}}} e^{i \Phi_{\text {probe }}(x)}, \\
F_{\text {pump }}(t) & =e^{-\frac{t^{2}}{\sigma_{t}^{2}}}, \\
\Phi_{\text {pump }}(x) & =-k_{0}\left(R_{z}-\sqrt{R_{z}^{2}-\left(x+x_{0}\right)^{2}}+\sin \theta\left(x+x_{0}\right)\right), \\
\Phi_{\text {probe }}(x) & =-k_{0}\left(R_{z}-\sqrt{R_{z}^{2}-\left(x-x_{0}\right)^{2}}-\sin \theta\left(x-x_{0}\right)\right), \\
F_{\text {probe }}(t) & =e^{-\frac{t^{2}}{\sigma_{t}^{2}}} \mathrm{e}^{i \Delta \omega t}, \\
\sigma_{x} & =\sigma_{y}=\sigma_{0} \sqrt{1+\frac{z_{0}^{2}}{z_{r}^{2}}}, \\
R_{z} & =z_{0}\left(1+\frac{z_{r}^{2}}{z_{0}^{2}}\right), z_{r}=\frac{\pi \sigma_{0}^{2}}{\lambda_{0}}, x_{0}=-z_{0} \tan (\theta), \\
\mathcal{E}_{\text {pump }} & =\sqrt{\frac{2}{\pi} \frac{P_{\text {pump }}}{\sigma_{x} \sigma_{y}}}, P_{\text {pump }}=\sqrt{\frac{2}{\pi}} \frac{E_{\text {pump }}}{\sigma_{t}}, \\
\mathcal{E}_{\text {probe }} & =\sqrt{\frac{2}{\pi} \frac{P_{\text {probe }}}{\sigma_{x} \sigma_{y}}, P_{\text {probe }}=\sqrt{\frac{2}{\pi}} \frac{E_{\text {probe }}}{\sigma_{t}},}
\end{aligned}
$$

where $R_{z}$ is the curvature radius and $z_{r}$ is the Rayleigh length. The initial conditions were chosen to be as close as possible to the experimental ones. The pump beam waist $\sigma_{0}$ is $40 \mu \mathrm{m}(34 \mu \mathrm{m})$ in the HOK (PG) model case, $\theta=3^{\circ}, \lambda_{0}=790 \mathrm{~nm}, z_{0}=-5 \mathrm{~mm}$, and $\sigma_{t}=85$ ss. In the two last equations, $E_{\text {pump }}\left(E_{\text {probe }}\right)$ is the pump (probe) energy and $P_{\text {pump }}\left(P_{\text {pump }}\right)$ is the pump (probe) peak power. Note that the probe energy was kept constant in all the simulations to $E_{\text {probe }}=0.8 \mu \mathrm{J}$ in order to match the experiment. The frequency detuning between the pump and probe pulse is $\Delta \omega$.

At the end of the propagation $(z=10 \mathrm{~mm})$, the probe beam does not overlap the pump beam anymore allowing the filtering of the former. The pure heterodyne signal obtained at a pump-probe delay $\tau$ is then evaluated for each spatial point by using a Jones matrix formalism describing the whole experimental detection setup and integrated over the entire spatiotemporal probe profile.

Note that, unlike the model developed in Ref. [3], the $3 \mathrm{D}+1$ model intrinsically embeds the plasma grating effect without the need to add its contribution in the model. For comparison with the full model, which takes into account both plasma grating and $\mathrm{HOK}$, the $\mathrm{HOK}$ model without plasma grating is obtained by setting the ionization cross-section $\sigma_{N}$ to 0 , while the PG model is obtained by setting the HOK indices to 0 .

Finally, one has to emphasize that, since the plasma grating model relies on the ionization process, the latter has to be modeled as good as possible. Such a modelling, which remains a challenging task, has been the subject of numerous theoretical works 35 38. In particular, it has been shown [39] that the ionization model first developed by Perelomov Popov and Terent'ev (PPT) reproduces well the experimental measurements performed in conditions close to the ones used in the present paper. However, the use of such a model leads to a dramatic increase of the computing-time and becomes prohibitive in $3 \mathrm{D}+1$ calculations when systematic studies have to be performed like in the present work. Nevertheless, PPT ionization rates can be accurately fitted by an effective multiphoton law (with a nonlinearity $\mathrm{N}=7.5$ ) in the in, tensity range $40-100 \mathrm{TW} / \mathrm{cm}^{2}$ [31], as shown in Fig. 2. Such a nonlinearity, better suited for intensive $3 \mathrm{D}+1$ calculations than the PPT formulation, has been confirmed by recent experimental measurements 40,41$]$.

\section{RESULTS AND DISCUSSION}

\section{A. One-color measurements}

\section{Fourier-transform limited (FTL) pulse}

The one-color pump-probe measurements have been conducted with $790 \mathrm{~nm}$ pump and probe beam both delivered by a Ti:Sapphire amplifier without using the NOPA system presented in Fig. 1. The birefringence signal recorded in argon with a heterodyne detection is shown with black circles in Fig. 3 for different input energies of the pump beam. All the experimental data are reported with the same scaling factor enabling thus a direct comparison between the different energies used. Each data point represents an averaging over 200 laser shots with a temporal sampling of $10 \mathrm{fs}$. In order to avoid significant nonlinear propagation effects, like intensity clamping, the gas pressure in the static cell was reduced to a low value reconcilable with an acceptable signal-to-noise ratio. As in the previous experiments [1, 5], the compression of the laser pulses at the interaction region was checked by maximizing the signal. The result obtained at $30 \mu \mathrm{J}$ [Fig. 3 (a)] is representative of the linear regime. It is featured by a linear variation of the signal near and below this energy and a temporal shape consistent with a birefringence $\Delta n$ associated with a pulse profile of the 100 fs-pump pulse. For laser energies close to and higher than $120 \mu \mathrm{J}$ [Fig. 3 (c)] the linear dependence of the signal on the pump energy is lifted and sign inversion is observed. The signal shown in Fig. 3(c) is typical of this inversion and has been already reported in atomic or molecular gas species close to 

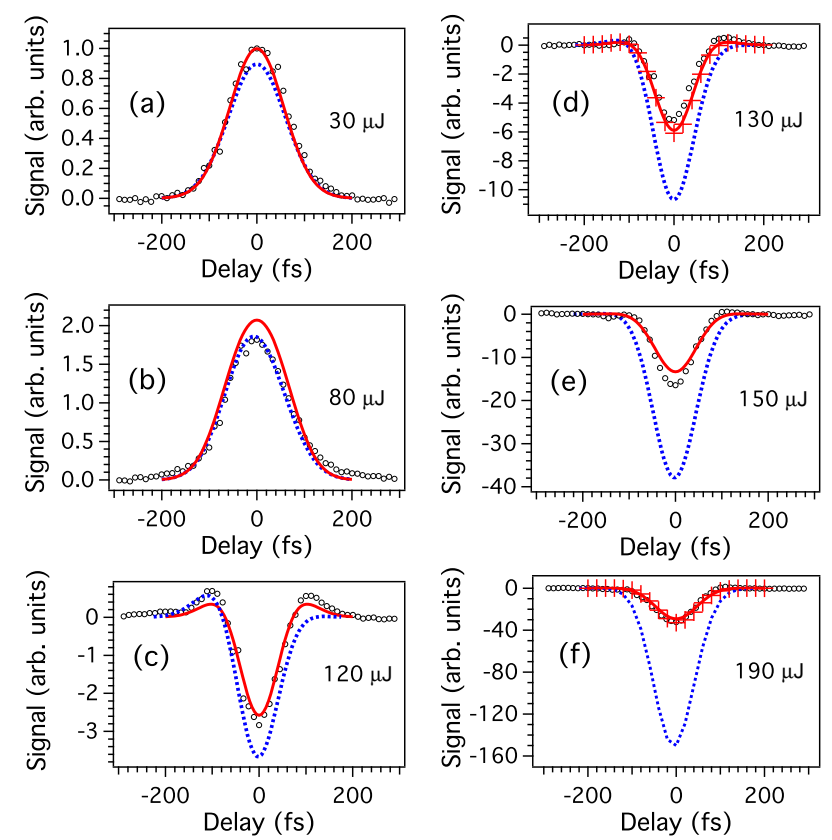

FIG. 3: (Color online) Pump energy dependence of the heterodyned birefringence signals (black circles) recorded in argon at a static pressure of (a-c) 400 and (d-f) 100 mbar versus the pump-probe delay. Data are compared to the $3 \mathrm{D}+1 \mathrm{nu}-$ merical simulations based on PG (blue doted curves), pure HOK model (red solid curves), and full HOK + PG model (red crosses). The Fourier-transform limited (FTL) pulse duration is $100 \mathrm{fs}$ and the wavelength of the pump and probe is $790 \mathrm{~nm}$. The beam waist in the PG model is reduced by $17 \%$ compared to the measured value (see text). It is noted that all curves have been centered at 0 pump-probe delay for comparison. The peak pump intensities $I_{\text {pump }}$ (expressed in $\mathrm{TW} / \mathrm{cm}^{2}$ ) evaluated with numerical simulations are $I_{\text {pump }}(\mathrm{HOK}) \simeq 0.37 \times E_{\text {pump }}$ and $I_{\text {pump }}(\mathrm{PG}) \simeq 0.52 \times E_{\text {pump }}$, where $E_{\text {pump }}$ is the pump energy expressed in $\mu \mathrm{J}$.

$25 \mathrm{TW} / \mathrm{cm}^{2}$ [1, 2, 5]. At higher energy values, the signal is dominated by a negative, rather symmetric, temporal profile reaching a minimum value close to the zero delay.

A comparison between the $3 \mathrm{D}+1$ numerical simulations and the experimental data is also presented in Fig. 3. The results based on the HOK model alone, i.e., without accounting for the ionization process, are shown with red solid curves. As shown the data are fairly well reproduced by the HOK model using the Kerr indices measured in Refs. [1, 2]. Since the model does not account for ionization, the negative part of the signal results from the negative high-order Kerr terms $n_{4}$ and $n_{8}$ [see Eqs. [6] and 7 that are the dominant contributions to the nonlinear refractive index above $120 \mu \mathrm{J}$. The influence of the ionization, and hence the contribution of the plasma grating to the birefringence measurements, has been assessed by calculating the theoretical signals without HOK. Because the ionization rate of Ar predicts a balance of the

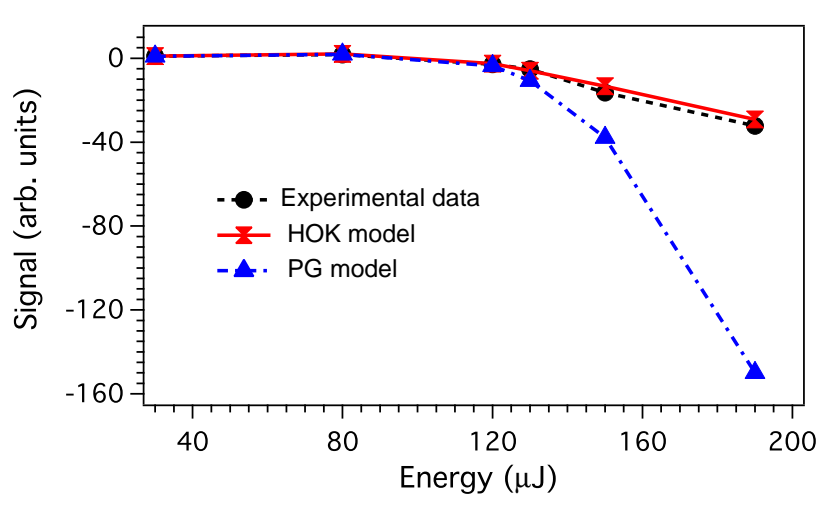

FIG. 4: (Color online) Energy dependence of the birefringence signal (see Fig. 3) measured near the zero pump-probe delay compared to both models.

positive Kerr $\left(n_{2}\right)$ by the PG effect beyond the energy employed in the experiment, the simulations have been performed with a beam waist decreased by $17 \%$ with respect to the measured value so as to allow a comparison with the experimental data. It should be emphasized that this adjustment does not impact the energy dependence of the signal. The results are shown with blue doted curves. Comparison between experimental data and PG model clearly indicates that the latter fails to reproduce the nonlinear dependence of the observed effect above the inversion. This is shown also in Fig. 4 where the dependence of the recorded signal on the laser energy is depicted along with the corresponding numerical simulations results for both models. Under the present experimental conditions, the ionization rate of Argon calculated in Fig. 2 approximately scales like the seventh power of the intensity between 40 and $100 \mathrm{TW} / \mathrm{cm}^{2}$ [31, 41] and is negligible for lower intensities. The magnitude of the PG effect being proportional to the ionization rate requires that the resulting birefringence obeys the same intensity dependence [3, 27]. On the contrary, the birefringence signal above $120 \mu \mathrm{J}$ changes like the fourth power of the applied energy, which is properly captured by the HOK model with $n_{8}$ being the dominant term at large intensity. Simulations including both HOK and PG effect have also been implemented using the experimental parameters of Fig. 3. No significant difference with the pure HOK model was observed as shown for instance in Figs. 3 (d) and (f). Finally, Fig. 5 shows that the intensity clamping resulting from nonlinear propagation effect is insignificant (less than 5\%), even in the PG model where the intensity is larger than in the HOK simulations. The previous results confirm that under the present conditions the PG acts as a marginal term in the present birefringence experiment. 


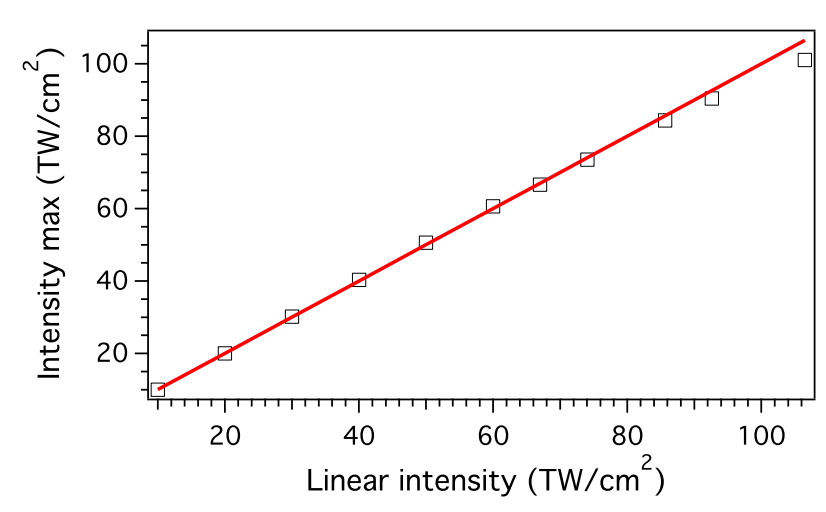

FIG. 5: (Color online) Peak intensity (black squares) calculated with the help of $3 \mathrm{D}+1$ simulations in the case of the PG model as a function of the intensity expected in the linear propagation regime. The linear regime (red solid curve) is shown to help the eye.

\section{Chirped pulse}

The birefringence induced by the plasma grating results from the accumulation of electrons produced all along the pulse duration. Due to its delayed nature, the negative phase shift of the PG combined with the positive $n_{2}$ Kerr index produces an asymmetric signal around the intensity of inversion where both effect counteract each other [3, 42]. No significant asymmetric profile was observed neither in the previous experiment [1, 5] nor in the present one [see for instance Fig. 3 (c)]. However, it was recently suggested that the absence of asymmetry could be the result of the the PG effect when considering temporally chirped pulses [42]. Although all our experiments so far were performed for compressed pump and probe beams, a set of data recorded with chirped pump and probe pulses around the region of inversion are compared to numerical simulations in Fig. 6. The linear chirp parameter has been characterized by SPIDER [Figs. 6 (a-c)] and autocorrelation [Figs. 6] (d, e)] measurements. It is noticed that $\pm 2150 \mathrm{fs}^{2}$ corresponds to a measured pulse duration of $160 \mathrm{fs}$. In order to compensate for the intensity drop introduced by the pulse stretching compared with the FTL pulse, the energy of the chirped pulse has been increased so as to recover the signal near the inversion region. The structural shape of the signals slightly differ with each other due to the high sensitivity of the effect with respect to the intensity. Nevertheless, one can see that the symmetry of the data is well conserved even for the largest chirp employed. On the contrary, the simulations presented in the same figure show that the structural shape of the PG signal is sensitive to the chirp [42]. Although, near the inversion the FTL-pulse signal is featured by an asymmetric shape (see Fig. 6 (b)], the latter can be reduced when using large chirps as shown for instance in Figs. 6] (d, e). The fact that the symmetry of the observed signals
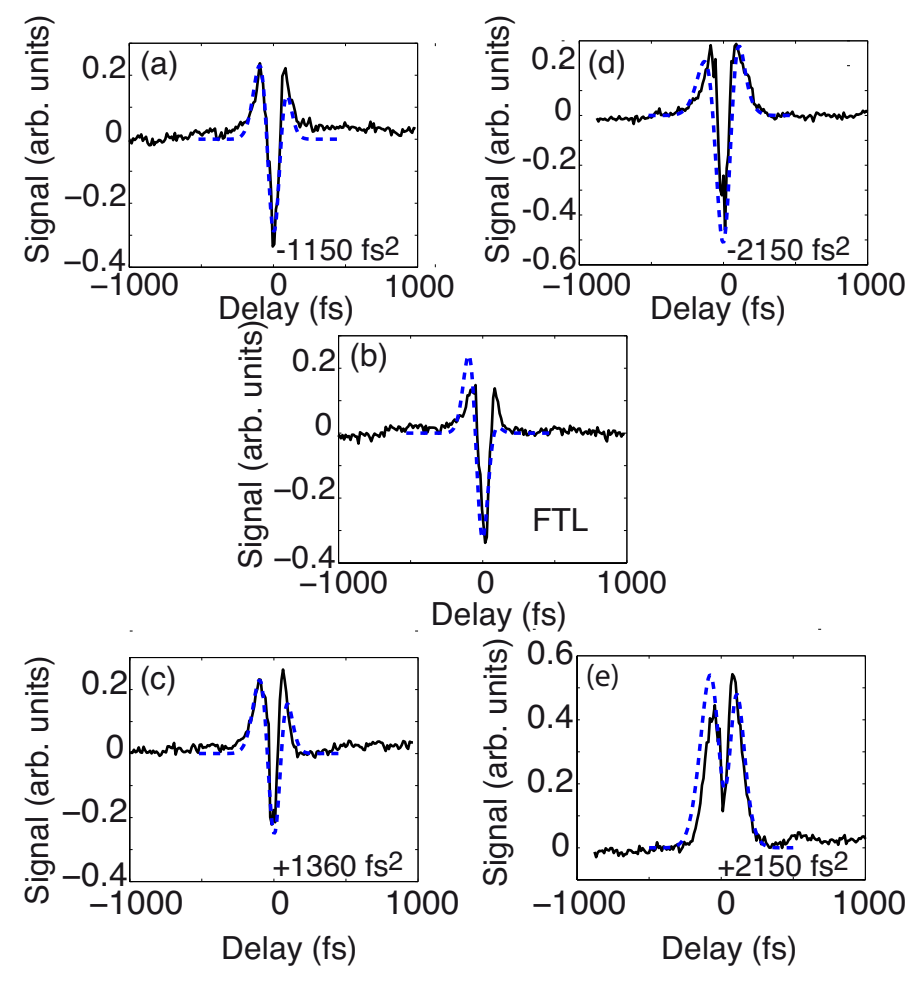

FIG. 6: (Color online) Comparison between heterodyned birefringence signals (black solid curves) recorded with (b) Fourier-transform limited (FTL) pulses and chirped pulses with different linear chirps: (a) $-1155 \mathrm{fs}^{2}$, (c) $+1360 \mathrm{fs}^{2}$, (d) $-2150 \mathrm{fs}^{2}$, and (e) $+2150 \mathrm{fs}^{2}$. Numerical simulations (blue dashed curves) based on the plasma-grating effect.

is preserved while the pulses are chirped and also that the HOK model does not predict any symmetry reversal of the signal allows to exclude the chirp as a possible artifact from our measurements. Finally, it should be pointed that large chirps, as those responsible for symmetric PG signal [42], lead at constant energy level to a dramatic drop of intensity and consequently to a significant decrease of the birefringence signal above the inversion threshold. This along with a temporal broadening of the signal could have been straightly noticed in the previous studies [1, 5] so that any unforeseen significant chirp would have been corrected.

\section{B. Two-color measurements}

Recently, a spectral analysis of the nonlinear polarization calculated with time-dependent Schrödinger equation [23] revealed that the saturation and inversion of the nonlinear refractive index mainly occurs within the bandwidth of the pump pulse in agreement with a recent observation [43]. This is consistent with single-color pumpprobe experiment, which exhibits refractive index saturation and inversion [1, 5, 43], and two-color experiments 

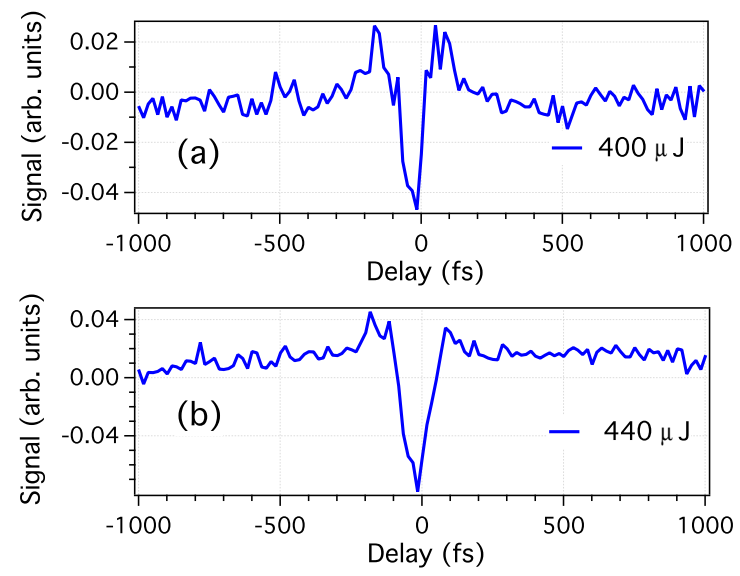

FIG. 7: (Color online) Two-color pump-probe heterodyne birefringent signals recorded in 100 mbar of argon with a pump input energy of (a) 400 and (b) $440 \mu \mathrm{J}$.

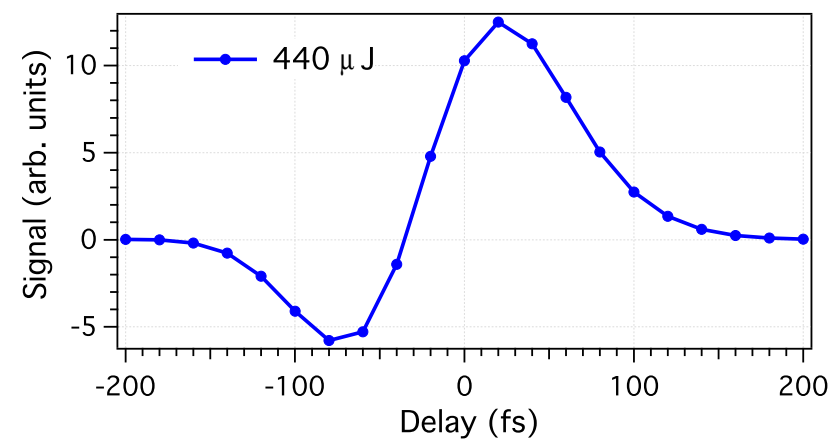

FIG. 8: (Color online) Two-color simulation of the plasma grating-induced birefringence for the input energy and pressure corresponding to Fig. 7(b).

using non overlapping spectra that do not [26, 43, 44]. However, the results presented in this section show that the sign inversion can also be produced with two-color non-overlapping spectra provided that much larger intensity is used compared to the one-color scheme.

The two-color studies have been performed with a 100 fs probe beam delivered by a non-collinear optical parametric amplifier (NOPA) inserted in the probe beam line, as shown in Fig. 1. The data of Fig. (7)have been recorded for a pump and probe wavelength set at 790 and $740 \mathrm{~nm}$, respectively. The birefringence signals presented in this figure are similar to those of Figs. 3 (c-d) or Fig. 6 (b), except that the energy had to be increased by a factor of $\sim 3$ in order to observe the inverted signal. So far this is the first observation of negative birefringence observed with non degenerate frequencies. Experiments performed with a larger detuning of the probe frequency, e.g., with $650 \mathrm{~nm}$ probe wavelength, did not reveal any inversion of the signal up to $800 \mu \mathrm{J}$.

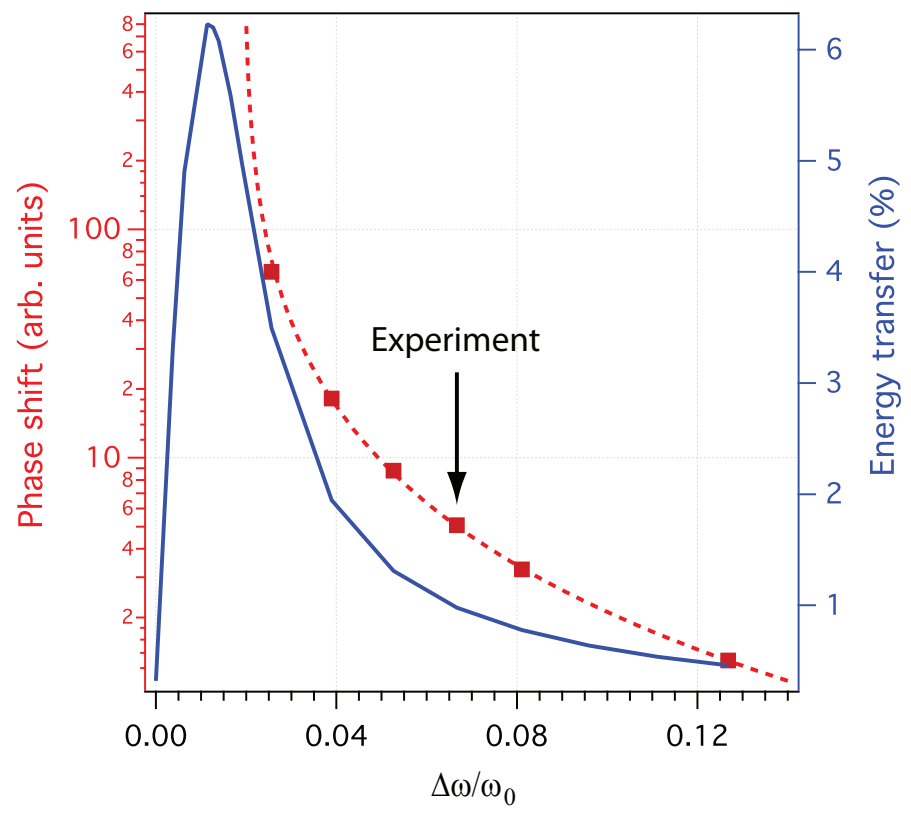

FIG. 9: (Color online) Plasma grating phase shift calculated numerically (left scale, red squares), its associated fit $\propto 1 /\left(\alpha+\beta \Delta \omega^{2}\right)$ (red dashed curve), and energy transfer (right scale, blue solid curve) calculated as a function of the frequency difference $\Delta \omega$ between the pump and probe beam. The arrow indicates the frequency difference of the experiment.

To point out the influence of the plasma grating in the two-color experiment, a 3D+1 numerical simulation corresponding to the experimental conditions of Fig. 7 was performed without HOK terms, but, as in the one-color case, with a beam waist reduction of $17 \%$. The result shown in Fig. 8 reveals that the PG effect also occurs for non degenerate frequencies, but it is much reduced, therefore larger energies are required for its observation. This is an unexpected result considering that as relying on optical interference, the PG effect is unlikely to take place with two-color non-overlapping spectra [42, 43]. In order to explain this apparent contradiction, let us recall that in two-color optical grating the difference between the two beam frequencies leads to a transverse shift of the optical fringes during the pulse. When the frequency detuning exceeds the pulse bandwidth, the spatial shift covers a distance larger than the fringe spacing of the optical grating during the pulse duration. Since ionization occurs within the whole pulse duration, the accumulated charges combined with the spatial shift of the optical grating should lead to the obliteration of the fringe contrast at the end of the pulse. However, rare gas ionization at $790 \mathrm{~nm}$ is a highly nonlinear process which mainly occurs around the maximum of the pulse, over a temporal window much shorter than its temporal width. During this short time interval, the contrast can be maintained even for frequency differences exceeding the pulse bandwidths. The frequency difference enabling a contrasted 


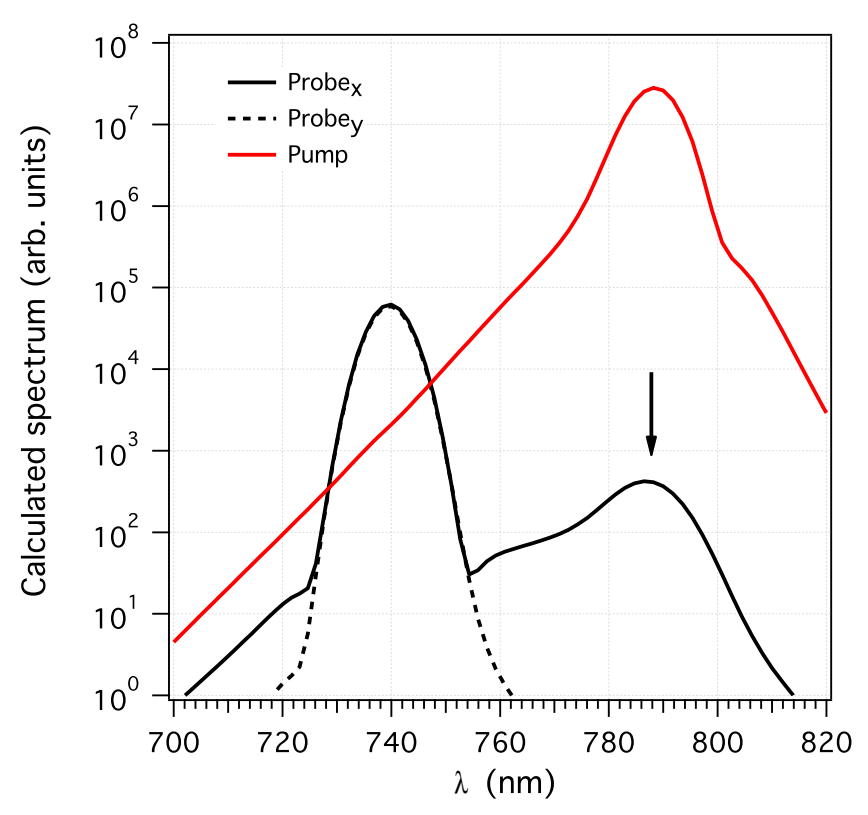

FIG. 10: (Color online) Polarization resolved pump and probe spectrum calculated after propagation though the Ar medium. The subscripts $x$ and $y$ refer to the polarization direction of the fields. Same conditions as in Fig. 8

grating is therefore inversely proportional to the ionization window. Figure 9 shows the birefringence resulting from the PG effect calculated for different pump-probe frequency differences $\Delta \omega$. The effect is well fitted by a $1 /\left(\alpha+\beta \Delta \omega^{2}\right)$ law as shown in Fig. 9 .

As in the one-color experiment, we can see that the birefringence signal of the two-color experiment does not match the calculated PG effect. In particular, a salient difference is observed between the temporal profiles. The pure PG effect in the non-degenerate frequencies case generates negative dispersion-like temporal shape centered on the zero delay, with a minimum (maximum) located at negative (positive) delay. When combined with the positive $n_{2}$ Kerr contribution, it results in a birefringence effect with a temporal profile such as in Fig. 8. which is totally inconsistent with the present observation. It was checked numerically that this disagreement could not result from a residual chirp of the pump and/or the probe beam. Finally, according to our numerical results, the high energies used in the measurements of Fig. 7 lead to the clamping of the pump intensity by a factor of $\sim 2$. Nevertheless, since our numerical simulations take into account the clamping phenomenon, the difference between PG model predictions and the experimental results cannot be assigned to the later.

A plasma grating can also be responsible for energy transfer between the pump and probe beam in two-color experiments [45], or in one-color chirped-pulse experiments [46]. It is known that the phase shift produced by the two-beam coupling is maximized for degenerate

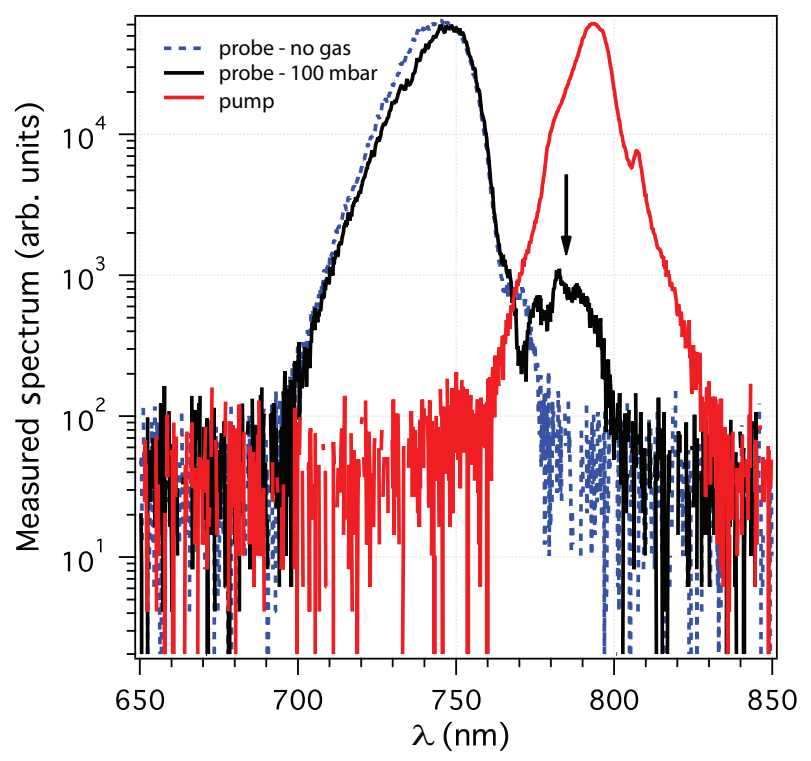

FIG. 11: (Color online) Normalized spectra measured at the medium exit. The pump energy is $440 \mu \mathrm{J}$.

frequencies [4]. On the contrary, the energy transfer does not reach its optimal value for degenerate frequencies but for a particular frequency detuning that depends on the characteristic time needed for building up the grating through ionization. Because of the spatial and temporal modulation of the grating, energy flow occurs from the pump to the probe beam, and vice versa depending on the sign of $\Delta \omega$. Both result in an energy exchange around the pump and probe frequency. For $\omega_{\text {probe }}>\omega_{\text {pump }}$, the simulations of Fig. 10 show that the energy flow is directed toward the probe beam, as evidenced by the energy bump centered around $790 \mathrm{~nm}$ and indicated by an arrow on the probe spectrum. This $\sim 1 \%$ of energy transfer is confirmed by the observation. Figure 11] shows the probe beam spectrum recorded with and without gas. Because the probe- to pump-energy ratio is less that $10^{-3}$, the relatively low energy loss experienced by the pump is not noticeable on the pump spectrum.

It is emphasized that our pump-probe measurements are not affected by neither polarization dependent energy transfer nor dichroïsm experienced by the probe as long as the ratio between the two polarization components remains close to unity. More particularly, using the Jones matrix formalism and assuming that the probe electric field at the end of the interaction has the general form

$$
\begin{aligned}
& E_{x}=\sqrt{T} \mathrm{e}^{i \Phi} \\
& E_{y}=1,
\end{aligned}
$$

where $\Phi$ is the nonlinear phase difference between the two polarization axes and $T$ is the intensity transmission along the $x$ dimension ( $T$ can be either lower than unity in the case of losses or higher than unity in the case of gain), one can show that the full heterodyne signal measured with the present technique is given by 


$$
S_{\text {heterodyne }} \propto 2 \sqrt{T} \sin \Psi_{\text {L.O. }} \sin \Phi,
$$

where $\Psi_{\text {L.O. }}$ is the phase of the local oscillator introduced in Eq. 2.

According to our 3D numerical model the energy transfer, as predicted by the PG model, is reproduced well from our experimental results. However as mentioned before, the latter effect unambiguously cannot solely describe the imparted phase to the probe beam, i.e. the birefringence signal, as shown from the comparison of figures 7 and 8.

\section{CONCLUSION}

Concluding, the active debate concerning the impact of the plasma grating (PG) effect on the nonlinear dynamics of optically induced birefringence is revisited by a systematic and thorough study in argon gas. New experimental data are presented at two different configurations concerning the laser wavelength, i.e., the degenerate and the non-degenerate case and the results are compared to both HOK and PG models with the help of full $3 \mathrm{D}+1$ numerical simulations.

In the degenerate configuration, the dependence of the induced birefringence on the power and the chirp of the laser pulse was examined. The power dependence measurements confirmed the sign inversion of the signal above a specific energy threshold and showed that for laser intensities well above the inversion it is proportional to the fourth power of the applied intensity, in full agreement with the HOK model. On the contrary, the PG effect is not adequate to account totally for the reported observations under the applied experimental conditions. In respect with the dependence on the chirp the symmetric peak profiles of the recorded signals for both chirped and FTL pulses further corroborate to this conclusion.

In the two non-degenerate beams configuration, it was shown, for the first time, that a saturation and a sign inversion of the nonlinear refractive index also take place. This sign inversion occurs at about 3 times higher intensity than in the degenerate case suggesting that the high-order indices are frequency-dependent. Moreover, the predicted birefringence profile from the PG model is found inconsistent with the recorded one while the predicted energy transfer (1\%) is in close resemblance with the experimental findings, making thus the impact of $\mathrm{PG}$ marginal also in this case. At this point it is emphasized that, besides its failure to reproduce our results, $\mathrm{PG}$ effect is still valid in the non-degenerate regime. The proposed interpretation for its occurrence is based on the fact that atomic ionization is a very fast process and takes place within a short time window around the peak intensity of the laser pulse. Thus, even in a non-degenerate beams scheme, it can be shown that despite the transverse shift of the optical fringes during the pulse duration, a contrasted grating remains inducing in turn a phase shift. Finally, albeit all the above experimental and theoretical data confirm the validity of using the HOK as an appropriate approach to the atomic response at moderate laser intensities, we do not overlook the fact that further studies, in both theory and experiment, are needed so as to provide the full physical mechanism behind it.
[1] V. Loriot, E. Hertz, O. Faucher, and B. Lavorel, Opt. Express 17, 13429 (2009).

[2] V. Loriot, E. Hertz, O. Faucher, and B. Lavorel, Opt. Express 18, 3011 (2010).

[3] J. K. Wahlstrand and H. M. Milchberg, Opt. Lett. 36, 3822 (2011).

[4] R. W. Boyd, Nonlinear Optics (Academic Press, Boston, 2008), 3rd ed.

[5] V. Loriot, P. Béjot, W. Ettoumi, Y. Petit, J. Kasparian, S. Henin, E. Hertz, B. Lavorel, O. Faucher, and J. P. Wolf, Laser Phys. 21, 1319 (2011).

[6] H. Stapelfeldt and T. Seideman, Rev. Mod. Phys. 75, 543 (2003).

[7] T. Seideman and E. Hamilton, Adv. At. Mol. Opt. Phys. 52, 289 (2006).

[8] V. Kumarappan, S. S. Viftrup, L. Holmegaard, C. Z. Bisgaard, and H. Stapelfeldt, Phys. Scr. 76, C63 (2007).

[9] P. Béjot, B. E. Schmidt, J. Kasparian, J. P. Wolf, and F. Legaré, Physical Review A 81, 063828 (2010).

[10] B. E. Schmidt, P. Béjot, M. Giguere, A. D. Shiner, C. Trallero-Herrero, E. Bisson, J. Kasparian, J.-P. Wolf, D. M. Villeneuve, J.-C. Kieffer, et al., Appl. Phys. Lett.
96, 121109 (2010).

[11] A. Couairon and A. Mysyrowicz, Phys. Rep. 441, 47 (2007).

[12] L. Bergé, S. Skupin, R. Nuter, J. Kasparian, and J.-P. Wolf, Rep. Prog. Phys. 70, 1633 (2007).

[13] S. Chin, S. Hosseini, W. Liu, Q. Luo, F. Theberge, N. Akozbek, A. Becker, V. Kandidov, O. Kosareva, and H. Schroeder, Can. J. Phys. 83, 863 (2005).

[14] P. Béjot, J. Kasparian, S. Henin, V. Loriot, T. Vieillard, E. Hertz, O. Faucher, B. Lavorel, and J. P. Wolf, Phys. Rev. Lett. 104, 103903 (2010).

[15] P. Béjot, E. Hertz, J. Kasparian, B. Lavorel, J. P. Wolf, and O. Faucher, Phys. Rev. Lett. 106, 243902 (2011).

[16] P. Polynkin, M. Kolesik, E. M. Wright, and J. V. Moloney, Phys. Rev. Lett. 106, 153902 (2011).

[17] P. Kano, M. Brio, and J. Moloney, Comm. Math. Sci. 4, 53 (2006).

[18] M. Nurhuda, A. Suda, and K. Midorikawa, Phys. Rev. A 66, 041802 (2002).

[19] M. Nurhuda, A. Suda, and K. Midorikawa, New J. Phys. 10, 053006 (2008).

[20] A. Teleki, E. M. Wright, and M. Kolesik, Phys. Rev. A 
82, 065801 (2010).

[21] E. Volkova, A. Popov, and O. Tikhonova, JETP Lett. 94, 519 (2011).

[22] E. Lorin, S. Chelkowski, E. Zaoui, and A. Bandrauk, Physica D 241, 1059 (2012).

[23] P. Béjot, E. Cormier, E. Hertz, B. Lavorel, J. Kasparian, J. P. Wolf, and O. Faucher, Phys. Rev. Lett. 110, 043902 (2013).

[24] C. Kohler, R. Guichard, E. Lorin, S. Chelkowski, A. Bandrauk, L. Bergé, and S. Skupin, Phys. Rev. A 87, 043811 (2013).

[25] C. Brée, A. Demircan, and G. Steinmeyer, Phys. Rev. Lett. 106, 183902 (2011).

[26] J. K. Wahlstrand, Y. H. Cheng, and H. M. Milchberg, Phys. Rev. Lett. 109, 113904 (2012).

[27] J. K. Wahlstrand, Y. H. Cheng, and H. M. Milchberg, Phys. Rev. A 85, 043820 (2012).

[28] B. Lavorel, O. Faucher, M. Morgen, and R. Chaux, J. Raman Spectrosc. 31, 77 (2000).

[29] V. Loriot, P. Tzallas, E. P. Benis, E. Hertz, B. Lavorel, D. Charalambidis, and O. Faucher, J. Phys. B. 40, 2503 (2007).

[30] M. Kolesik and J. Moloney, Phys. Rev. E 70, 036604 (2004).

[31] J. Kasparian, R. Sauerbrey, and S. Chin, Appl. Phys. B 71, 877 (2000).

[32] V. Loriot, E. Hertz, B. Lavorel, and O. Faucher, J. Phys. B 41, 015604 (2008).

[33] J. Arabat and J. Etchepare, J. Opt. Soc. Am. B 10, 2377 (1993).

[34] G. Stegeman, D. G. Papazoglou, R. Boyd, and S. Tzortzakis, Opt. Express 19, 6387 (2011).

[35] L. Keldysh, SOVIET PHYSICS JETP 20, 1307 (1965).

[36] A. Perelomov, V. Popov, and M. Terent'ev, SOVIET
PHYSICS JETP 23, 924 (1966).

[37] M. Ammosov, N. Delone, and V. Krainov, Zh. Eksp. Teor. Fiz. 91, 2008 (1986).

[38] S. Popruzhenko, V. Mur, V. Popov, and D. Bauer, Phys. Rev. Lett. 101, 193003 (2008).

[39] A. Talebpour, J. Yang, and S. Chin, Opt. Comm. 163, 29 (1999).

[40] E. Wells, M. DeWitt, and R. Jones, Phys. Rev. A 66, 113409 (2002).

[41] F. Billard, P. Béjot, E. Hertz, B. Lavorel, and O. Faucher, Phys. Rev. A 88, 013854 (2013).

[42] J. K. Wahlstrand, J. H. Odhner, E. T. McCole, Y. H. Cheng, J. P. Palastro, R. J. Levis, and H. M. Milchberg, Phys. Rev. A 87, 053801 (2013).

[43] J. H. Odhner, D. A. Romanov, E. T. McCole, J. K. Wahlstrand, H. M. Milchberg, and R. J. Levis, Phys. Rev. Lett. 109, 065003 (2012).

[44] J. K. Wahlstrand, Y. H. Cheng, Y. H. Chen, and H. M. Milchberg, Phys. Rev. Lett. 107, 103901 (2011).

[45] X. Yang, J. Wu, Y. Tong, L. Ding, Z. Xu, and H. Zeng, Appl. Phys. Lett. 97, 071108 (2010).

[46] Y. Liu, M. Durand, S. Chen, A. Houard, B. Prade, B. Forestier, and A. Mysyrowicz, Phys. Rev. Lett. 105, 055003 (2010).

\section{Acknowledgments}

This work was supported by the Conseil Régional de Bourgogne (PARI and FABER programs), the CNRS, and the Labex ACTION program (contract ANR-11LABX-01-01). P.B. thanks the CRI-CCUB for CPU loan on its multiprocessor server. 